\title{
Preliminary Evaluation of Morphological and Pomological Characters to Illustrate Genetic Diversity of Apricots (Prunus armeniaca L.) in Afghanistan
}

\author{
Hedayatullah Salari, Alam Khan Samim, Saifuddin Ahadi, Sayed Ahmad Etemadi
}

\begin{abstract}
Afghanistan assumed to be a part of the diversity center of Central Asian group of apricots but due to lack of proper collection, precise detection and documentation of distinct variability, the apricot diversity in the country is under unknown and unprecedented threat. This study, therefore, was run in Badam-Bagh research station for two consecutive seasons (2016-2017) to assess 28 various characters of 20 native Afghan apricot accessions. Results showed a high variability among studied genotypes. Leaf and fruit characteristics such as leaf blade length, leaf blade width, fruit weight, kernel weight, mesocarp percentage, kernel bitterness and fruit quality like total soluble solids, $\mathrm{pH}$, and titratable acidity, skin color and fruit shape which subsequently determines customer appeal and marketability, displayed huge variation. Based on fruit weight, there were two groups of apricot genotypes; small fruits $(<37 \mathrm{~g})$ and large fruits $(>37 \mathrm{~g})$. Accessions with large fruits embrace: "Amiri”, "Bid-Mushk", "Hassan-Khili", "Jibraelli", "Koorpa", "Qandak", "Pir-Naqshi", "SeyedAbadi", "Salari-Sorkh", and "Saqi". Most genotypes were early flowering and intermediately ripening in nature. Kernels of only "Walayati" accession had strong bitterness and remained were sweet-kernelled. The mesocarps of most accessions were very weakly adhered to stones. PCA showed $\mathbf{8 6 . 1 5 \%}$ of total variance among genotypes to be due to first seven components. UPGMA cluster analysis separated 20 accessions into 2 main groups (13 accessions in the first and 7 accessions in the second groups). The results exhibited that morphological characters of leaf and fruit and pomological characters are of immense importance in fast and easy description of apricot genotypes. This is per se the first study on genetic diversity of apricot accessions performed in Afghanistan. The results would provide opportunity for apricot breeders to select accessions with elite and premium characters in terms of marketability and adaptability for particular growing sites.
\end{abstract}

Index Terms - Apricot, germplasm, pomological traits, variability.

\section{INTRODUCTION}

In Afghanistan, by volume and cultivating area, apricot is the fifth most important deciduous fruit used as fresh and dried [4]. Apricot production occupies 23,890 hectares of the country land with $55,175 \mathrm{~kg}$ per hectare average yield

Published on September 19, 2020.

Hedayatullah Salari, Horticulture Department, Paktia University, Afghanistan.

(e-mail: h.salari786@ gmail.com)

Alam Khan Samim, Horticulture Department, Paktia University, Afghanistan.

(e-mail: alamkhantotakhel ${ }^{@}$ gmail.com)

Saifuddin Ahadi, Germplasm Curator, National Fruit Germplasm, Kabul, Afghanistan.

Sayed Ahmad Etemadi, Field Horticulturist, Badambagh Fruit Research Station, Kabul, Afghanistan. and 13,1816 tons total annual production [13]. Apricot species is characterized by a large genetic variability organized in phylum in which a strong interaction exists between cultivars and areas of cultivation.

For a century, apricot collections have been increasing year by year by new selections, which are very important to raise and to improve production and quality of apricots. In 1969, Kostina classified apricots into four eco-geographical groups: the Central Asian group, the Dzhungar-Zailij group, the Irano-Caucasian group, and the European group [19]. Afghanistan presumes to be one of the diversity center of apricot where rich diversity exists [5]. Vavilov has also introduced Afghanistan as an apricot indigenous country that is included in the Central Asian Diversity Center [20]. In Afghanistan, huge number of apricot varieties are grown under different agro-climatic conditions. The apricot diversity in Afghanistan is very high as there are at least 128 apricot accessions in National Fruit Collection of the country from which about 92 apricot accessions are listed in the Afghan National Fruit Collection catalogue.

Fruit germplasm plays a prominent role in breeding of crops. Breeding programs can introduce a variety of apricots adapted to different climatic conditions and suitable for market requirements in terms of quality and quantity. But explaining the phenological, morphological, and pomological characteristics of the cultivars is one of the basic needs of a breeding program that has not yet been carried out for apricot germplasm, and the risk of extinction of these genetic resources is increasing if not documented and managed.

Although more studies have been done on apricot cultivars of European center [16] and Irano-Caucasian center [7], and they have resulted in leading apricot cultivars that are being grown in most parts of the world today but studies on apricot cultivars in Central Asia (of which Afghanistan is also a part) are very limited. Therefore, the management of genetic resources (germplasm), which includes the collection, precise detection, and documentation of extant variability, is very important for the conservation, breeding programs and commercialization of apricot cultivars in the region.

Therefore, the present study was undertaken to evaluate genetic diversity in apricot cultivars grown throughout the country. Although there are reports on apricot cultivars description, they are either not scientific or are limited to a few varieties. This will be the first report about apricot cultivars grown in Afghanistan and may provide the foundation for effective management and sustainable use of apricot germplasm in the country. 


\section{MATERIALS AND METHODS}

\section{A. Site, Design and Plant Materials}

This study was conducted during two consecutive years 2016 and 2017. Twenty ex situ reputable apricot native accessions (native to the country) were selected from national fruit collection of Badam-Bagh Research Station, Kabul, Afghanistan. The apricot fruit collection was established in 2008, having 138 ex situ accessions collected from previously marked exemplar (in situ) trees around the country. They were raised from budding of each accession scion on to domestic apricot seedlings and planted at an experimental farm following Randomized Block Design (RBD) with three replications. The three plants of each twenty genotypes were planted at $4.5 \times 3 \mathrm{~m}$ spacing. Twenty apricot cultivars were: ("Amiri", "Baghal Sorkh", "BidMushk", "Hassan khili”, "Jibraeli”, "koorpa”, "Maidani”, "Qandak", "Pir-Naqshi”, "Sayed Abadi”, "Salari-Sorkh", "Saqi", "Senjidi", "Shakarpara", "Shakarpara Sorkh", "Shakarpara Wakili”, "Shirin Bnoo", "Timori”, "Walayati”, and "Waziri") and their specifications are presented in Table 1.

\section{B. Measurements}

Twenty eight characters were recorded from 20 accessions/genotypes of apricot during the study. The tree habit, leaf and fruit characteristics of all selected genotypes were recorded based on standard DUS guidelines on apricot. The UPOV apricot descriptor list was used [2], [29]. There were two types of characters. The first characters were those assessed objectively as nominal or categorical scales: flowering date, ripening date, kernel bitterness, fruit flesh firmness, adherence of flesh to stone, pubescence fruit surface, position of stigma in relation to anther, fresh color, skin color was objectively assessed (Table 1).

The second type were characters measured by definite units and measurement tools: tree height $(\mathrm{TH}, \mathrm{m})$, flower diameter (FD, mm), leaf blade length (LBL, mm), leaf blade width (LBW, mm), ratio between leaf blade length and width (LBL/LBW), petiole length (PL, mm), ratio between leaf blade length and petiole length (LBL/PL), petiole thickness $(\mathrm{PT}, \mathrm{mm})$, fruit length $(\mathrm{FL}, \mathrm{mm})$, fruit lateral width (FLW, mm), fruit ventral width (FVW, mm), fruit weight $(\mathrm{FW}, \mathrm{g})$, stone weight $(\mathrm{SW}, \mathrm{g})$, ratio between fruit and stone weights (FW/SW), fruit shape index (FSI), mesocarp percentage (MP, \%), total soluble solids (TSS, oBrix), fruit $\mathrm{pH}$, and Titratable acidity (TA, \%).

A total of 30 fruits and stones were sampled per each genotype for physical, chemical, and sensorial analyses. Fruit, stone and kernel weight was measured with the help of digital scale ET-1201 (Iskra, Kranj, China) (range of measurement $0.01-120.00 \mathrm{~g}$, precision $\pm 0.01 \mathrm{~g}$ ) and expressed in gram $(\mathrm{g})$. Fruit and leaf dimensions were measured with the help of an electronic digital caliper 150 $\mathrm{mm}$ (Mitutoyo Corporation, Japan). Fruit flesh firmness was determined using organoleptic test as soft, medium firm and very soft. Fruit characteristics were observed at maturity stage, when fruit was ready for harvest and leaf characteristics were recorded from fully developed leaves of the middle third of current season's shoot.

Fruit shape index (FSI) was calculated as $\mathrm{FSI}=(\mathrm{W}+\mathrm{L}) / 2 \mathrm{~L}$ where; $\mathrm{W}=$ fruit weight, $\mathrm{L}=$ fruit length and $\mathrm{T}=$ fruit lateral width [33]. Mesocarp percentage (MP) was calculated as $[(\mathrm{FW}-\mathrm{SW}) / \mathrm{FW} * 100]$ where; $\mathrm{FW}=$ fruit weight, $\mathrm{SW}=$ stone weight [14].

\section{Data analysis}

All data are means of two years. Analysis of variance (ANOVA), Pearson's correlation coefficients, Fisher's least significant difference $(\mathrm{LSD})$ test $(\mathrm{P}<0.05)$ were calculated using SPSS 20 software (SPSS, Inc., Chicago, USA). Clustering of genotypes into similarity groups was done using the method of UPGA (Unweighted Pair Group Average) with SPSS 20 (SPSS, Inc., Chicago, USA). Principal component analysis (PCA) was performed to evaluate relationships among variables and any possible cultivar groupings based on similar properties by using SPSS 20 (SPSS, Inc., Chicago, USA). In the result section, from ANOVA tables, only higher and lower values reported not mean comparison.

\section{RESUlTS}

\section{A. Tree height and Flower Diameter}

Tree height $(\mathrm{TH})$ had high variability among apricot accessions, range from minimum $(2.53 \mathrm{~m})$ in acc. "Amiri" to maximum $(5.50 \mathrm{~m})$ in acc. "Waziri" with mean TH of $4.48 \mathrm{~m}$ (Tables I and III).

Flower diameter (FD) showed huge variations in studied genotypes, ranged from minimum FD $(17.83 \mathrm{~mm})$ in acc. "Shakarpara-Wakili" to maximum FD $(37.67 \mathrm{~mm})$ in acc. "Pir-Naqshi" with mean FD of $27.78 \mathrm{~mm}$ (Tables II \& III). With regard to Table 1, there were eight accessions with stigmas above anthers ["Baghal-Sorkh", "Gibraelli", "Maidani", "Senjidi", "Shakarpara", "Shakarpara-Sorkh", "Shakarpara-Wakili", and "Shirin-Bano"] but in remained accessions the stigamas were below anthers. Furthermore, from 20 accessions, 15 accessions were early flowering that would be late-spring frost sensitive (Table I).

\section{B. Leaf Characters}

Results of the study showed high variations in relation to various leaf dimensions among Afghan apricot native cultivars (Tables II, and III). Huge variation was observed in LBL of 20 genotypes, ranged from minimum $(62.06 \mathrm{~mm})$ in acc. "Waziri" to maximum $(93.33 \mathrm{~mm})$ in acc. "Timuri" with mean LBL of $62.06 \mathrm{~mm}$. LBW was varied from minimum $(29.67 \mathrm{~mm})$ in acc. "Shakarpara-Sorkh" to maximum $(83.50 \mathrm{~mm})$ in acc. "Maidani" with mean LBW of $59.80 \mathrm{~mm}$. As seen in Tables 2 and 3, the ratio between BL and BW was highly different among studied genotypes which ranged from minimum (0.67) in acc "ShakarparWakili" to maximum ratio (1.32) in acc. "ShakarparaSorkh" and mean BL/BW of 1.06. The PL among 20 genotypes was highly varied from minimum $(17.17 \mathrm{~mm})$ in acc. "Senjidi" to maximum $(49.67 \mathrm{~mm})$ in acc. "Koorpa" with mean PL of $30.43 \mathrm{~mm}$. PT varied from minimum $(6.38 \mathrm{~mm})$ in acc. "Koorpa" to maximum $(12.85 \mathrm{~mm})$ in acc. "Timuri" with mean PT (9.14 mm) among 20 apricot genotypes. Among 20 genotypes, there was huge variation in relation to BL/PL ranged from minimum (1.56) in acc. "Salari-Sorkh" to maximum (3.13) in acc. "Pir-Naqshi" and mean BL/PL of 2.10 (Tables II, III). 


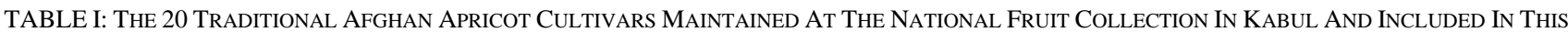
STUdy With THEIR ORIGin, QUALITATIVE AND PHONOLOGICAL CHARACTERS

\begin{tabular}{|c|c|c|c|c|c|c|c|c|c|c|c|}
\hline Code & Cultivars & Origin & Skin color & Flesh color & $\begin{array}{c}\text { Flowering } \\
\text { date }^{1}\end{array}$ & $\begin{array}{c}\text { Ripening } \\
\text { date }^{1}\end{array}$ & $\begin{array}{c}\text { Fruit } \\
\text { flesh } \\
\text { firmness }\end{array}$ & $\begin{array}{c}\text { Kernel } \\
\text { bitterness }^{3}\end{array}$ & $\begin{array}{c}\text { Adherence } \\
\text { of flesh to } \\
\text { stone }^{4}\end{array}$ & $\begin{array}{c}\text { Pubescence } \\
\text { of fruit } \\
\text { surface }^{5}\end{array}$ & $\begin{array}{l}\text { Position of } \\
\text { Stigma to } \\
\text { relation to } \\
\text { another }\end{array}$ \\
\hline AFG0247 & Amiri & Wardak (Jaghato) & yellow green & Creamy & $\mathrm{E}$ & I & Soft & $a / w$ & vw & $\mathrm{P}$ & Below \\
\hline AFG4025 & Baghal Sorkh & Herat /Guzara & yellow green & light orange & $\mathrm{I}$ & I & Medium & $a / w$ & $\mathrm{w}$ & $\mathrm{p}$ & above \\
\hline AFG0246 & Bid-Mushk & Wardak (Jaghato) & light orange & light orange & I & I & Soft & $\mathrm{a} / \mathrm{w}$ & vw & $\mathrm{p}$ & Below \\
\hline AFG0825 & Hassan khili & $\begin{array}{c}\text { Wardak/Saed } \\
\text { Abad }\end{array}$ & yellow green & Creamy & I & I & Medium & $\mathrm{a} / \mathrm{w}$ & vw & $\mathrm{p}$ & Below \\
\hline AFG4018 & Jibraeli & Herat/Guzara & yellow green & creamy & $\mathrm{E}$ & $\mathrm{E}$ & Medium & $\mathrm{a} / \mathrm{w}$ & vw & $\mathrm{p}$ & above \\
\hline AFG0243 & koorpa & Wardak (Jaghato) & yellow green & creamy & $\mathrm{E}$ & $\mathrm{I}$ & Soft & $\mathrm{a} / \mathrm{w}$ & vw & a & Below \\
\hline AFG0290 & Maidani & $\begin{array}{c}\text { Laghman } \\
\text { (Mehtarlam) }\end{array}$ & yellow green & light orange & I & I & Medium & $\mathrm{a} / \mathrm{w}$ & vw & $\mathrm{a}$ & above \\
\hline AFG5001 & Qandak & Logar (Khushiy) & orange red & creamy & $\mathrm{E}$ & $\mathrm{I}$ & Medium & $\mathrm{a} / \mathrm{w}$ & $\mathrm{m}$ & $\mathrm{a}$ & Below \\
\hline AFG0292 & Pir-Naqshi & Balkh (Khulm) & orange red & creamy & $\mathrm{E}$ & $\mathrm{E}$ & Medium & $\mathrm{a} / \mathrm{w}$ & vw & $\mathrm{p}$ & Below \\
\hline AFG0827 & Sayed Abadi & $\begin{array}{l}\text { Wardak (Saed } \\
\text { Abad) }\end{array}$ & yellow green & creamy & $\mathrm{E}$ & $\mathrm{E}$ & Soft & $\mathrm{a} / \mathrm{w}$ & vw & $\mathrm{p}$ & Below \\
\hline AFG0824 & Salari-Sorkh & $\begin{array}{c}\text { Wardak (Saed } \\
\text { Abad) }\end{array}$ & yellow green & $\begin{array}{l}\text { medium } \\
\text { orange }\end{array}$ & $\mathrm{E}$ & I & very soft & $\mathrm{a} / \mathrm{w}$ & w & $\mathrm{p}$ & Below \\
\hline AFG50 02 & Saqi & $\begin{array}{l}\text { Bamiyan } \\
\text { (Kahmak) }\end{array}$ & $\begin{array}{l}\text { red over light } \\
\text { green }\end{array}$ & $\begin{array}{l}\text { medium } \\
\text { orange }\end{array}$ & $\mathrm{E}$ & I & Soft & $\mathrm{a} / \mathrm{w}$ & $\mathrm{m}$ & a & Below \\
\hline AFG0249 & Senjidi & Wardak (Jaghato) & yellowish & creamy & $\mathrm{E}$ & I & Medium & $\mathrm{a} / \mathrm{w}$ & vw & $\mathrm{a}$ & above \\
\hline AFG0373 & Shakarpara & $\begin{array}{l}\text { Kandahar } \\
\text { (Arghan) }\end{array}$ & yellow green & whitish green & $\mathrm{E}$ & I & Medium & $\mathrm{a} / \mathrm{w}$ & $\mathrm{a} / \mathrm{vw}$ & $\mathrm{p}$ & above \\
\hline AFG0250 & $\begin{array}{c}\text { Shakarpara } \\
\text { Sorkh }\end{array}$ & Wardak (Jaghato) & yellow green & Creamy & $\mathrm{E}$ & I & Soft & $\mathrm{a} / \mathrm{w}$ & w & $\mathrm{p}$ & above \\
\hline AFG0275 & $\begin{array}{l}\text { Shakarpara } \\
\text { Wakili }\end{array}$ & Wardak (Nerkh) & $\begin{array}{l}\text { orange red over } \\
\text { light green }\end{array}$ & white & $\mathrm{E}$ & I & Medium & $\mathrm{a} / \mathrm{w}$ & vw & $\mathrm{p}$ & above \\
\hline AFG0331 & Shirin Bnoo & $\begin{array}{c}\text { Kanduz } \\
\text { (Charakar) }\end{array}$ & $\begin{array}{l}\text { pink over light } \\
\text { green }\end{array}$ & white & I & I & Medium & $\mathrm{a} / \mathrm{w}$ & $\mathrm{m}$ & $\mathrm{p}$ & above \\
\hline AFG0823 & Timori & $\begin{array}{c}\text { Wardak (Saed } \\
\text { Abad) }\end{array}$ & $\begin{array}{c}\text { purple over } \\
\text { medium yellow }\end{array}$ & light orange & $\mathrm{E}$ & I & Soft & $\mathrm{a} / \mathrm{w}$ & vw & $\mathrm{p}$ & Below \\
\hline AFG0208 & Walayati & $\begin{array}{l}\text { Nangarhar } \\
\text { (Jalalabad) }\end{array}$ & orange & light orange & $\mathrm{E}$ & $\mathrm{E}$ & Soft & $\mathrm{s}($ strong) & vw & $\mathrm{a}$ & Below \\
\hline AFG4017 & Waziri & Herat (Guzara) & yellow green & creamy & $\mathrm{E}$ & $\mathrm{E}$ & Medium & $a / w$ & vw & $\mathrm{a}$ & Below \\
\hline
\end{tabular}

${ }^{1}$ Flowering and Ripening date: E: early; I: intermediate; and L: late.

${ }^{2}$ Firmness. S: soft; M: medium; and VS: very soft.

${ }^{3}$ Kernel bitterness: a/w: absent or very weak; w: weak; m: medium; and s: strong.

${ }^{4}$ Adherence of flesh to stone: a/vw: absent or very weak; w: weak; and s: strong.

5 Pubescence of fruit surface: a: absent; $p$ : present.

TABLE II: TREE HEIGHT AND LEAF CHARACTERS RECORDED ON 20 AFGHAN APRICOT CULTIVARS

\begin{tabular}{|c|c|c|c|c|c|c|c|c|}
\hline \multirow[b]{2}{*}{ Cultivars } & \multirow[b]{2}{*}{$\begin{array}{c}\text { Tree height } \\
(\mathrm{m})\end{array}$} & \multirow[b]{2}{*}{$\begin{array}{c}\text { Flower diameter } \\
(\mathbf{m m})\end{array}$} & \multicolumn{6}{|c|}{ Leaf characters } \\
\hline & & & $\begin{array}{c}\text { Leaf blade } \\
\text { length }(\mathrm{mm})\end{array}$ & $\begin{array}{l}\text { Leaf blade } \\
\text { width }(\mathrm{mm})\end{array}$ & $\begin{array}{c}\text { Ratio of blade } \\
\text { length to blade } \\
\text { width }\end{array}$ & $\begin{array}{c}\text { Petiole } \\
\text { length }(\mathbf{m m})\end{array}$ & $\begin{array}{c}\text { Ratio of Blade } \\
\text { length to petiole } \\
\text { length }\end{array}$ & $\begin{array}{c}\text { Petiole } \\
\text { thickness } \\
(\mathbf{m m})\end{array}$ \\
\hline AFG0247Amiri & $2.53 \mathrm{~d}$ & $27.33 \mathrm{bcd}$ & $55.50 \mathrm{c}-\mathrm{e}$ & $56.00 \mathrm{~d}$ & $0.99 \mathrm{~d}-\mathrm{g}$ & $33.33 \mathrm{de}$ & $1.67 \mathrm{e}$ & $9.35 \mathrm{bc}$ \\
\hline AFG4025Baghal Sorkh & $4.21 \mathrm{bc}$ & $36.50 \mathrm{a}$ & $91.00 \mathrm{a}$ & $77.00 \mathrm{~b}$ & $1.18 \mathrm{a}-\mathrm{e}$ & $36.17 \mathrm{~cd}$ & $2.52 \mathrm{bc}$ & $9.80 \mathrm{bc}$ \\
\hline AFG0246Bid-Mushk & $4.25 \mathrm{bc}$ & $36.33 \mathrm{a}$ & $92.55 \mathrm{a}$ & $76.67 \mathrm{~b}$ & $1.20 \mathrm{a}-\mathrm{d}$ & $48.67 \mathrm{ab}$ & $1.90 \mathrm{de}$ & 7.07 ef \\
\hline AFG0825Hassan khili & $5.15 \mathrm{ab}$ & $28.67 \mathrm{bc}$ & $43.17 \mathrm{f}-\mathrm{h}$ & $39.83 \mathrm{e}$ & $1.08 \mathrm{~b}-\mathrm{g}$ & $19.50 \mathrm{f}$ & $2.21 \mathrm{~b}-\mathrm{e}$ & $9.52 \mathrm{bc}$ \\
\hline AFG0243koorpa & $4.06 \mathrm{bc}$ & 21.00 efg & $93.00 \mathrm{a}$ & $81.50 \mathrm{ab}$ & $1.14 \mathrm{a}-\mathrm{f}$ & $49.67 \mathrm{a}$ & $1.87 \mathrm{de}$ & $6.38 \mathrm{f}$ \\
\hline AFG0290Maidani & $4.73 \mathrm{a}-\mathrm{c}$ & $19.33 \mathrm{fg}$ & $90.50 \mathrm{a}$ & $83.50 \mathrm{a}$ & $1.08 \mathrm{~b}-\mathrm{g}$ & $38.83 \mathrm{~cd}$ & $2.33 \mathrm{~b}-\mathrm{d}$ & $9.88 \mathrm{bc}$ \\
\hline AFG5001Qandak & $4.45 \mathrm{a}-\mathrm{c}$ & $28.17 \mathrm{bc}$ & $38.83 \mathrm{gh}$ & $40.00 \mathrm{e}$ & $0.97 \mathrm{e}-\mathrm{g}$ & $19.33 \mathrm{f}$ & $2.01 \mathrm{c}-\mathrm{e}$ & $6.68 \mathrm{f}$ \\
\hline AFG0292Pir Noor Shi & $4.08 \mathrm{bc}$ & $37.67 \mathrm{a}$ & $91.28 \mathrm{a}$ & $80.17 \mathrm{ab}$ & $1.14 \mathrm{a}-\mathrm{f}$ & $29.17 \mathrm{e}$ & $3.13 \mathrm{a}$ & $9.93 \mathrm{bc}$ \\
\hline AFG0827Sayed Abadi & $4.16 \mathrm{bc}$ & $31.33 \mathrm{~b}$ & $54.17 \mathrm{~d}-\mathrm{f}$ & $79.67 \mathrm{ab}$ & $0.68 \mathrm{~h}$ & $19.50 \mathrm{f}$ & $2.78 \mathrm{ab}$ & $12.37 \mathrm{a}$ \\
\hline AFG0824Salari-Sorkh & $4.23 \mathrm{bc}$ & $28.17 \mathrm{bc}$ & $62.83 \mathrm{~b}-\mathrm{d}$ & $61.33 \mathrm{~d}$ & $1.05 \mathrm{c}-\mathrm{g}$ & $39.67 \mathrm{~cd}$ & $1.58 \mathrm{e}$ & $9.42 \mathrm{bc}$ \\
\hline AFG5002Saqi & $4.33 \mathrm{bc}$ & $26.50 \mathrm{bcd}$ & $45.33 \mathrm{e}-\mathrm{g}$ & $37.17 \mathrm{e}$ & $1.22 \mathrm{a}-\mathrm{c}$ & $18.83 \mathrm{f}$ & $2.41 \mathrm{~b}-\mathrm{d}$ & $9.17 \mathrm{bc}$ \\
\hline AFG0249Senjidi & $4.96 \mathrm{a}-\mathrm{c}$ & $36.33 \mathrm{a}$ & $40.83 \mathrm{gh}$ & 34.17 ef & $1.19 \mathrm{a}-\mathrm{d}$ & $17.17 \mathrm{f}$ & $2.38 \mathrm{~b}-\mathrm{d}$ & $8.93 \mathrm{~cd}$ \\
\hline AFG0250Shakarpara Sorkh & $4.86 \mathrm{a}-\mathrm{c}$ & $19.17 \mathrm{fg}$ & $39.00 \mathrm{gh}$ & $29.67 \mathrm{f}$ & $1.32 \mathrm{a}$ & $18.17 \mathrm{f}$ & $2.15 \mathrm{~b}-\mathrm{e}$ & 7.36 ef \\
\hline AFG0275Shakarpara Wakili & $5.03 \mathrm{a}-\mathrm{c}$ & $17.83 \mathrm{~g}$ & $54.83 \mathrm{c}-\mathrm{f}$ & $81.50 \mathrm{ab}$ & $0.67 \mathrm{~h}$ & $33.00 \mathrm{de}$ & $1.66 \mathrm{e}$ & $10.28 \mathrm{~b}$ \\
\hline AFG0331Shirin Bnoo & $5.16 \mathrm{ab}$ & $27.33 \mathrm{bcd}$ & $39.50 \mathrm{gh}$ & $37.67 \mathrm{e}$ & $1.06 \mathrm{c}-\mathrm{g}$ & $18.50 \mathrm{f}$ & $2.14 \mathrm{c}-\mathrm{e}$ & $9.32 \mathrm{bc}$ \\
\hline AFG0823Timori & $4.11 \mathrm{bc}$ & $37.67 \mathrm{a}$ & $93.33 \mathrm{a}$ & $80.83 \mathrm{ab}$ & $1.16 \mathrm{a}-\mathrm{f}$ & $48.00 \mathrm{ab}$ & $1.94 \mathrm{c}-\mathrm{e}$ & $12.85 \mathrm{a}$ \\
\hline AFG0208Walayati & $5.06 \mathrm{a}-\mathrm{c}$ & $25.33 \mathrm{cde}$ & $66.33 \mathrm{bc}$ & $68.50 \mathrm{c}$ & $0.97 \mathrm{e}-\mathrm{g}$ & $42.50 \mathrm{bc}$ & $1.56 \mathrm{e}$ & $8.97 \mathrm{~cd}$ \\
\hline AFG4017Waziri & $5.50 \mathrm{a}$ & $23.17 \mathrm{def}$ & $33.50 \mathrm{~h}$ & 35.00 ef & $0.96 \mathrm{fg}$ & $17.83 \mathrm{f}$ & $1.88 \mathrm{de}$ & $10.17 \mathrm{~b}$ \\
\hline Significance & $* * *$ & $* * *$ & 0.0000 & 0.0000 & 0.0000 & 0.0000 & 0.0005 & 0.0000 \\
\hline Fisher's $L S D_{0.05}$ & 1.12 & 4.97 & 11.72 & 6.07 & 0.21 & 6.95 & 0.62 & 1.18 \\
\hline Coefficient of variation (\%) & 15.23 & 10.82 & 11.43 & 6.15 & 12.41 & 13.82 & 17.72 & 7.83 \\
\hline
\end{tabular}

The similar letter(s) within vertical columns indicate insignificant difference between means (LSD test, $P \leq 0.05$ ). 
TABLE III: Flower DiAmeter AND Pomological Characters Relate To 20 AfGHAN Apricot Cultivars

\begin{tabular}{|c|c|c|c|c|c|c|c|c|c|c|c|c|}
\hline \multirow[b]{2}{*}{ Code } & \multirow[b]{2}{*}{ Cultivar } & \multicolumn{6}{|c|}{ Fruit pomological characters } & \multicolumn{5}{|c|}{ Fruit quality characters } \\
\hline & & $\begin{array}{l}\text { Length } \\
(\mathrm{mm})\end{array}$ & $\begin{array}{c}\text { Lateral } \\
\text { width } \\
\text { (mm) }\end{array}$ & $\begin{array}{c}\text { Fruit } \\
\text { ventral } \\
\text { width }(\mathbf{m m})\end{array}$ & Weight (g) & $\begin{array}{c}\text { Stone } \\
\text { weight }(g)\end{array}$ & $\begin{array}{c}\text { Fruit-Stone } \\
\text { weight } \\
\text { ratio }\end{array}$ & $\begin{array}{l}\text { Fruit } \\
\text { Shape } \\
\text { Index }\end{array}$ & $\begin{array}{l}\text { Mesocarp } \\
\text { Percentage }\end{array}$ & $\begin{array}{c}\text { Total } \\
\text { Soluble } \\
\text { Solids } \\
\left({ }^{\circ} \text { Brix }\right)\end{array}$ & $\begin{array}{c}\text { Titratable } \\
\text { acidity }(\%)\end{array}$ & pH \\
\hline AFG0247 & Amiri & $46.67 \mathrm{a}-\mathrm{d}$ & $38.83 \mathrm{~cd}$ & $26.17 \mathrm{~h}$ & $48.22 \mathrm{a}-\mathrm{c}$ & $2.72 \mathrm{~cd}$ & $17.77 \mathrm{a}$ & $0.94 \mathrm{~b}-\mathrm{d}$ & $94.23 \mathrm{ab}$ & $1.09 \mathrm{f}-\mathrm{i}$ & $1.09 \mathrm{f}-\mathrm{i}$ & $4.09 \mathrm{~d}-\mathrm{g}$ \\
\hline AFG4025 & Baghal Sorkh & $43.17 \mathrm{~b}-\mathrm{f}$ & $36.00 \mathrm{~d}-\mathrm{g}$ & $44.83 \mathrm{a}-\mathrm{c}$ & $33.17 \mathrm{e}-\mathrm{g}$ & $3.30 \mathrm{~b}-\mathrm{d}$ & $10.17 \mathrm{de}$ & $0.85 \mathrm{c}-\mathrm{e}$ & $90.03 \mathrm{~d}-\mathrm{f}$ & $1.36 \mathrm{~b}-\mathrm{e}$ & $1.36 \mathrm{~b}-\mathrm{e}$ & $3.98 \mathrm{~g}$ \\
\hline AFG0246 & Bid-Mushk & $37.17 \mathrm{~d}-\mathrm{h}$ & $31.33 \mathrm{e}-\mathrm{g}$ & $41.67 \mathrm{~b}-\mathrm{f}$ & $42.83 \mathrm{c}-\mathrm{e}$ & $3.76 \mathrm{ab}$ & $11.57 \mathrm{~b}-\mathrm{e}$ & $1.01 \mathrm{~b}-\mathrm{d}$ & $91.13 \mathrm{~b}-\mathrm{f}$ & $1.20 \mathrm{e}-\mathrm{h}$ & $1.20 \mathrm{e}-\mathrm{h}$ & $4.04 \mathrm{fg}$ \\
\hline AFG0825 & Hassan khili & $54.83 \mathrm{a}$ & $53.00 \mathrm{a}$ & $37.00 \mathrm{c}-\mathrm{f}$ & $54.83 \mathrm{ab}$ & $3.05 \mathrm{~cd}$ & $18.11 \mathrm{a}$ & $0.99 \mathrm{~b}-\mathrm{d}$ & $94.44 \mathrm{a}$ & $1.00 \mathrm{~g}-\mathrm{i}$ & $1.00 \mathrm{~g}-\mathrm{i}$ & $4.06 \mathrm{e}-\mathrm{g}$ \\
\hline AFG4018 & Jibraelli & $34.00 \mathrm{f}-\mathrm{h}$ & $34.67 \mathrm{~d}-\mathrm{g}$ & $44.83 \mathrm{a}-\mathrm{c}$ & $45.39 \mathrm{~b}-\mathrm{d}$ & $2.78 \mathrm{~cd}$ & $16.16 \mathrm{ab}$ & $1.21 \mathrm{ab}$ & $93.44 \mathrm{a}-\mathrm{c}$ & $1.51 \mathrm{~b}-\mathrm{d}$ & $1.51 \mathrm{~b}-\mathrm{d}$ & $4.49 \mathrm{a}-\mathrm{c}$ \\
\hline AFG0243 & koorpa & $34.00 \mathrm{f}-\mathrm{h}$ & $31.17 \mathrm{e}-\mathrm{g}$ & $36.83 \mathrm{~d}-\mathrm{f}$ & $39.92 \mathrm{c}-\mathrm{e}$ & $3.90 \mathrm{ab}$ & $10.27 \mathrm{de}$ & $1.12 \mathrm{a}-\mathrm{c}$ & $90.21 \mathrm{c}-\mathrm{f}$ & $1.45 \mathrm{~b}-\mathrm{e}$ & $1.45 \mathrm{~b}-\mathrm{e}$ & $3.97 \mathrm{~g}$ \\
\hline AFG0290 & Maidani & $31.83 \mathrm{gh}$ & $36.50 \mathrm{~d}-\mathrm{f}$ & $36.17 \mathrm{~d}-\mathrm{f}$ & $35.43 \mathrm{~d}-\mathrm{f}$ & $3.29 \mathrm{~b}-\mathrm{d}$ & $10.73 \mathrm{c}-\mathrm{e}$ & $1.16 \mathrm{a}-\mathrm{c}$ & $90.40 \mathrm{c}-\mathrm{f}$ & $1.56 \mathrm{~b}$ & $1.56 \mathrm{~b}$ & $4.07 \mathrm{~d}-\mathrm{g}$ \\
\hline AFG5001 & Qandak & $45.83 \mathrm{a}-\mathrm{e}$ & $30.33 \mathrm{fg}$ & $42.33 b-f$ & $57.57 \mathrm{a}$ & $3.89 \mathrm{ab}$ & $15.31 \mathrm{a}-\mathrm{c}$ & $0.96 \mathrm{~b}-\mathrm{d}$ & $93.23 \mathrm{a}-\mathrm{c}$ & $1.08 \mathrm{f}-\mathrm{i}$ & $1.08 \mathrm{f}-\mathrm{i}$ & $4.59 \mathrm{ab}$ \\
\hline AFG0292 & Pir Noor Shi & $47.33 \mathrm{a}-\mathrm{d}$ & $35.67 \mathrm{~d}-\mathrm{g}$ & 43.17 a-e & $47.62 \mathrm{a}-\mathrm{c}$ & $2.63 \mathrm{~d}$ & $18.11 \mathrm{a}$ & $0.90 \mathrm{~b}-\mathrm{e}$ & $94.40 \mathrm{ab}$ & $1.86 \mathrm{a}$ & $1.86 \mathrm{a}$ & $4.04 \mathrm{fg}$ \\
\hline AFG0827 & Sayed Abadi & $40.83 \mathrm{c}-\mathrm{g}$ & $35.17 \mathrm{~d}-\mathrm{g}$ & $36.50 \mathrm{~d}-\mathrm{f}$ & $39.34 c-f$ & $3.34 \mathrm{bc}$ & $11.83 \mathrm{~b}-\mathrm{e}$ & $0.93 \mathrm{~b}-\mathrm{e}$ & $91.38 \mathrm{a}-\mathrm{f}$ & $1.30 \mathrm{c}-\mathrm{f}$ & $1.30 \mathrm{c}-\mathrm{f}$ & $4.04 \mathrm{fg}$ \\
\hline AFG0824 & Salari-Sorkh & $38.50 \mathrm{c}-\mathrm{h}$ & $47.50 \mathrm{ab}$ & $45.00 \mathrm{ab}$ & $57.35 \mathrm{a}$ & $4.13 \mathrm{a}$ & 13.97 a-e & $1.37 \mathrm{a}$ & 92.75 a-e & $1.25 \mathrm{~d}-\mathrm{g}$ & $1.25 \mathrm{~d}-\mathrm{g}$ & $4.02 \mathrm{fg}$ \\
\hline AFG5002 & Saqi & $43.83 \mathrm{~b}-\mathrm{f}$ & $30.00 \mathrm{fg}$ & 39.67 b-f & $38.74 \mathrm{c}-\mathrm{f}$ & $2.63 \mathrm{~d}$ & $14.71 \mathrm{a}-\mathrm{d}$ & $0.79 \mathrm{de}$ & $92.94 \mathrm{a}-\mathrm{d}$ & $1.42 \mathrm{~b}-\mathrm{e}$ & $1.42 \mathrm{~b}-\mathrm{e}$ & $4.33 \mathrm{~b}-\mathrm{d}$ \\
\hline AFG0249 & Senjidi & $53.50 \mathrm{ab}$ & $33.33 \mathrm{~d}-\mathrm{g}$ & $35.50 \mathrm{e}-\mathrm{g}$ & $28.77 \mathrm{f}-\mathrm{h}$ & $2.82 \mathrm{~cd}$ & $10.32 \mathrm{de}$ & $0.59 \mathrm{e}$ & 89.48 ef & $1.04 \mathrm{f}-\mathrm{i}$ & $1.04 \mathrm{f}-\mathrm{i}$ & $3.94 \mathrm{~g}$ \\
\hline AFG0373 & Shakarpara & $30.17 \mathrm{gh}$ & $31.67 \mathrm{e}-\mathrm{g}$ & $27.83 \mathrm{gh}$ & $20.52 \mathrm{~h}$ & $1.85 \mathrm{e}$ & $11.73 \mathrm{~b}-\mathrm{e}$ & $0.88 \mathrm{~b}-\mathrm{e}$ & $90.94 c-f$ & 0.94 hi & $0.94 \mathrm{hi}$ & $4.29 \mathrm{c}-\mathrm{f}$ \\
\hline AFG0250 & $\begin{array}{l}\text { Shakarpara } \\
\text { Sorkh }\end{array}$ & $29.50 \mathrm{~h}$ & $35.50 \mathrm{~d}-\mathrm{g}$ & $36.67 \mathrm{~d}-\mathrm{f}$ & $18.17 \mathrm{~h}$ & $1.82 \mathrm{e}$ & $10.31 \mathrm{de}$ & $0.91 \mathrm{~b}-\mathrm{e}$ & 89.98 d-f & $0.94 \mathrm{hi}$ & 0.94 hi & $4.46 \mathrm{a}-\mathrm{c}$ \\
\hline AFG0275 & $\begin{array}{l}\text { Shakarpara } \\
\text { Wakili }\end{array}$ & $30.33 \mathrm{gh}$ & $37.17 \mathrm{de}$ & $43.50 \mathrm{a}-\mathrm{d}$ & $20.37 \mathrm{~h}$ & $1.74 \mathrm{e}$ & $11.81 \mathrm{~b}-\mathrm{e}$ & $0.96 \mathrm{~b}-\mathrm{d}$ & $91.47 \mathrm{a}-\mathrm{f}$ & $0.96 \mathrm{hi}$ & 0.96 hi & $4.50 \mathrm{a}-\mathrm{c}$ \\
\hline AFG0331 & Shirin Bnoo & $35.00 \mathrm{e}-\mathrm{h}$ & $44.17 \mathrm{bc}$ & $40.00 \mathrm{~b}-\mathrm{f}$ & $20.06 \mathrm{~h}$ & $1.75 \mathrm{e}$ & $11.62 \mathrm{~b}-\mathrm{e}$ & $0.92 \mathrm{~b}-\mathrm{e}$ & $91.33 \mathrm{a}-\mathrm{f}$ & $0.92 \mathrm{i}$ & $0.92 \mathrm{i}$ & $4.64 \mathrm{a}$ \\
\hline AFG0823 & Timori & $49.00 \mathrm{a}-\mathrm{c}$ & $37.17 \mathrm{de}$ & $50.83 \mathrm{a}$ & $35.62 \mathrm{~d}-\mathrm{f}$ & $3.83 \mathrm{ab}$ & 9.33 ef & $0.77 \mathrm{de}$ & $89.15 \mathrm{f}$ & $1.52 \mathrm{bc}$ & $1.52 \mathrm{bc}$ & $4.14 \mathrm{~d}-\mathrm{g}$ \\
\hline AFG0208 & Walayati & $29.33 \mathrm{~h}$ & $30.33 \mathrm{fg}$ & $28.00 \mathrm{gh}$ & $19.72 \mathrm{~h}$ & $1.74 \mathrm{e}$ & 13.47 a-e & $0.86 \mathrm{c}-\mathrm{e}$ & $90.95 \mathrm{c}-\mathrm{f}$ & $0.96 \mathrm{hi}$ & $0.96 \mathrm{hi}$ & $4.15 \mathrm{~d}-\mathrm{g}$ \\
\hline AFG4017 & Waziri & $28.83 \mathrm{~h}$ & $29.67 \mathrm{~g}$ & $34.67 \mathrm{fg}$ & $22.84 \mathrm{gh}$ & $4.37 \mathrm{a}$ & $5.25 \mathrm{f}^{-}$ & $0.91 \mathrm{~b}-\mathrm{e}$ & $80.88 \mathrm{~g}$ & $1.02 \mathrm{~g}-\mathrm{i}$ & $1.02 \mathrm{~g}-\mathrm{i}$ & $4.33 \mathrm{~b}-\mathrm{z}$ \\
\hline \multicolumn{2}{|c|}{ Significance } & $* * *$ & $* * *$ & $* * *$ & $* * *$ & $* * *$ & $* *$ & $*$ & $* * *$ & $* * *$ & $* * *$ & *** \\
\hline \multirow{2}{*}{\multicolumn{2}{|c|}{ Fisher's $L S D_{0.05}$}} & 10.94 & 6.81 & 7.95 & 10.81 & 3.27 & 4.66 & 0.33 & 3.27 & 0.830 & 0.266 & 0.275 \\
\hline & & 16.89 & 11.46 & 12.48 & 18.01 & 2.17 & 22.35 & 21.23 & 2.17 & 3.99 & 13.23 & 3.96 \\
\hline
\end{tabular}

The similar letter(s) within vertical columns indicate insignificant difference between means (LSD test, $\mathrm{P} \leq 0.05$.

\section{Fruit Characters}

As shown in Table I, the dominant skin and flesh colors were yellow-green and creamy, respectively. The maximum, minimum and mean values of fruit characters of 20 different genotypes of Afghan apricot are presented in Tables II and IV. A high degree of variation was found in quantitative characteristics related to the fruit. Table II reveals that the FL had high variations among 20 genotypes. FL ranged from minimum $(28.83 \mathrm{~mm})$ in acc. "Waziri" to maximum $(54.83 \mathrm{~mm})$ in acc. "Hassan-Khili" with mean FL of 39.18 $\mathrm{mm}$. FLW was greatly varied among apricot genotypes ranged from minimum $(29.67 \mathrm{~mm})$ in acc. "Waziri" to maximum $(53.00 \mathrm{~mm})$ in acc. "Hassan-Khili" with mean FLW of $35.96 \mathrm{~mm}$. A high degree variation was found in FVW of 20 apricot genotypes, ranged from minimum $(26.17 \mathrm{~mm})$ in "Amiri" to maximum $(50.83 \mathrm{~mm})$ in acc. "Timuri" with mean FVW of $38.56 \mathrm{~mm}$. Great variations were found among FW of studied genotypes, varied from minimum $(18.17 \mathrm{~g})$ in acc. "Shakarpar-Sorkh" to maximum $(57.57 \mathrm{~g})$ in acc. "Qandak" with mean FW of $36.32 \mathrm{~g}$. SW was a highly variable character among 20 studied genotypes ranged from minimum (1.74g) in acc. "Shakarpara-Wakili" to maximum $(4.37 \mathrm{~g})$ in acc. "Waziri" with mean SW of $2.97 \mathrm{~g}$. The FW/SW had huge variation among 20 genotypes, varied from minimum (8.34) in acc. "Waziri" to maximum (33.83) in acc. "Hassan-Khili" with mean FW/SW of 14.86 (Tables II and IV).

\section{Fruit quality characters}

Fruit ripening of most accessions was occurred in midseason and fruits of five accessions namely "Gibraelli", "Pir-Naqshi", "Seyed-Abadi", "Walayati" and "Waziri", were early-ripening in nature (Table I). As seen in Table I, the fruits of only seven accession were non-pubescent the rest were all pubescent. FSI was highly variable for 20 studied genotypes, ranged from minimum (0.67) in acc. "Senjidi" to maximum (1.37) in acc. "Salari-Sorkh" with mean FSI of 1.06 (Table II and IV). With regard to FSI value, there is three main fruit shapes: [ovoid fruits: FSI $>1.00$; round fruits; FSI $\approx 1.00$; long fruits; FSI $<1.00]$. Data presented in Tables II and IV show great variation in MP among 20 studied genotypes with minimum (80.0\%) in acc. "Waziri" and maximum MP (94.4\%) in acc. "HassanKhili" and mean MP of $91.27 \%$. Mesocarps of 14 accessions were very weakly adhered to stones (Table I). Fruit $\mathrm{pH}$ had great variation among 20 apricot genotypes, ranged from minimum $\mathrm{pH}$ (3.94) in acc. "Senjidi" to maximum $\mathrm{pH}$ (4.64) in acc. "Shirin-Bano" with mean $\mathrm{pH}$ of 4.21. TA showed great variability among 20 genotypes, varied from minimum $(0.91 \%)$ in acc. "Shirin-Bano" to maximum $(1.86 \%)$ in acc. "Pir-Naqshi" with mean TA of $1.22 \%$. There was great variation in TSS, ranged from minimum (11.14 brix) in acc. "Salari-Sorkh" to maximum (14.13 brix) in acc. "Shakarpara-Wakili" with mean TSS of 12.60 brix degrees (Table II and IV). Mesocarp (flesh) firmness was very soft in acc. "Salari-Sorkh" and in the rest were from soft to medium (Table I). Kernels were found to be strongly bitter only in acc. "Walayati" while in case of remained accessions were found to be absent or weak (Table I). 
TABLE IV: Descriptive Statistics (Minimum, MaXimum, Standard DeViation, Range, MeAn, Standard ERror Of MeAn And Cv: CoefFicient

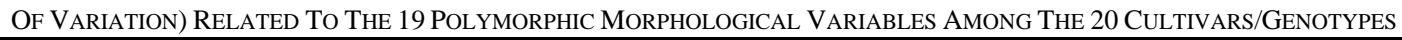

\begin{tabular}{|c|c|c|c|c|c|c|c|}
\hline Variables & Mean & Std. Error of Mean & Std. Deviation & Range & Minimum & Maximum & $\mathbf{C V}$ \\
\hline \multicolumn{8}{|l|}{ Tree } \\
\hline Tree height $(\mathrm{m})$ & 4.48 & 0.14 & 0.64 & 2.97 & 2.53 & 5.50 & 0.14 \\
\hline \multicolumn{8}{|l|}{ Leaf } \\
\hline Leaf blade length $(\mathrm{mm})$ & 62.06 & 4.99 & 22.30 & 59.83 & 33.50 & 93.33 & 0.24 \\
\hline Leaf blade width $(\mathrm{mm})$ & 59.80 & 4.74 & 21.22 & 53.83 & 29.67 & 83.50 & 0.21 \\
\hline Leaf Blade length:Blade width ratio & 1.06 & 0.04 & 0.17 & 0.65 & 0.67 & 1.32 & 0.17 \\
\hline Petiole length (mm) & 30.43 & 2.66 & 11.88 & 32.50 & 17.17 & 49.67 & 0.16 \\
\hline Leaf blade length:Petiole length ratio & 2.10 & 0.09 & 0.41 & 1.57 & 1.56 & 3.13 & 0.29 \\
\hline Petiole thickness (mm) & 9.14 & 0.38 & 1.69 & 6.47 & 6.38 & 12.85 & 0.57 \\
\hline \multicolumn{8}{|l|}{ Flower } \\
\hline Flower diameter $(\mathrm{mm})$ & 27.78 & 1.48 & 6.62 & 19.84 & 17.83 & 37.67 & 0.08 \\
\hline \multicolumn{8}{|l|}{ Fruit } \\
\hline Fruit length (mm) & 39.18 & 1.88 & 8.40 & 26.00 & 28.83 & 54.83 & 0.36 \\
\hline Fruit lateral width (mm) & 35.96 & 1.37 & 6.13 & 23.33 & 29.67 & 53.00 & 0.22 \\
\hline Fruit ventral width (mm) & 38.56 & 1.42 & 6.35 & 24.66 & 26.17 & 50.83 & 0.05 \\
\hline Fruit weight $(\mathrm{g})$ & 36.32 & 2.94 & 13.14 & 39.40 & 18.17 & 57.57 & 0.36 \\
\hline Stone weight $(\mathrm{g})$ & 2.97 & 0.19 & 0.86 & 2.63 & 1.74 & 4.37 & 0.35 \\
\hline Fruit:Stone weight ratio & 14.86 & 1.89 & 8.45 & 25.49 & 8.34 & 33.83 & 0.16 \\
\hline Mesocarp percentage $(\%)$ & 91.27 & 0.66 & 2.94 & 13.60 & 80.89 & 94.49 & 0.39 \\
\hline Total soluble solids (B) & 12.60 & 0.23 & 1.01 & 2.99 & 11.14 & 14.13 & 0.19 \\
\hline Titratable acidity $(\%)$ & 1.22 & 0.06 & 0.27 & 0.94 & 0.92 & 1.86 & 0.18 \\
\hline $\mathrm{pH}$ & 4.21 & 0.05 & 0.22 & 0.70 & 3.94 & 4.64 & 0.03 \\
\hline Fruit shape index & 1.06 & 0.04 & 0.17 & 0.64 & 0.67 & 1.31 & 0.17 \\
\hline
\end{tabular}

\section{E. Pearson Correlation Coefficients}

Bivariate correlation coefficients presented in Table V. Correlation between LBL and LBW was highly significant and positive $(\mathrm{r}=0.832)$ i.e. whilst between LBW and LBL/LBW was negative ( $r=-0.342)$. Correlation between PL and LBL was positive ( $\mathrm{r}=0.796)$ and between PL and LBW was positive $(r=0.758)$. Correlation coefficient between PL and ratio of $\mathrm{LBL} / \mathrm{PL}(\mathrm{r}=-0.419)$ was significantly negative while between LBL/PL and LBL/LBW was significantly positive $(\mathrm{r}=0.314)$. FD had a significant positive correlation with LBL $(r=0.347)$, PT $(r=0.288)$, ratio of $\mathrm{LBL} / \mathrm{PL}$ $(r=0.331)$. Fruit length was correlated, negatively with tree height $(\mathrm{r}=-0.369)$ and positively with $\mathrm{FD}(\mathrm{r}=0.565)$ and LBL/PL ( $r=0.345)$. Correlation of FVW was significantly positive with FD ( $r=0.328)$, LBL ( $r=0.333)$, LBW ( $r=0.333)$, PL ( $r=0.333)$, and FL ( $r=0.299)$; while in case of between FW and tree height was negative $(r=-0.447)$ and was positive with FD $(\mathrm{r}=0.358)$ and $\mathrm{FL}(\mathrm{r}=0.371)$. Correlation computations showed that SW was positively correlated with FD ( $\mathrm{r}=0.285)$, LBL ( $\mathrm{r}=0.281)$, FVW ( $\mathrm{r}=0.282)$ and FW $(r=0.512)$. These correlations show that with increasing FD, LBL, FVW and FW, SW accordingly increased. Correlations of FW/SW ratio with tree height $(\mathrm{r}=-0.322)$ and SW ( $\mathrm{r}=-0.306)$ were negative but were positive with FLW $(\mathrm{r}=0.257), \mathrm{FW}(\mathrm{r}=0.635)$ and FSI $(\mathrm{r}=0.303)$. The coefficients show that with increasing tree height, the ratio of FW/SW decreases i.e. vigor trees produce fruits with larger stones and, conversely, with increasing FW, FLW and FSI, FW/SW ratio also increases. Mesocarp percentage was correlated negatively with tree height $(\mathrm{r}=-0.364)$ and SW $(\mathrm{r}=-0.373)$ and positively with $\mathrm{FW}(\mathrm{r}=0.556)$ i.e. the higher the FW, the higher the MP, conversely, the higher the tree height or SW, the lower the MP.

The correlation between TSS and tree height was positive $(\mathrm{r}=0.299)$ while it was negative between TSS and FW ( $\mathrm{r}=-$ $0.267)$ or SW ( $r=-0.354)$. These coefficients mean that vigor trees produce fruits containing higher TSS than dwarf ones, while by increasing FW and SW, TSS decreases. There were negative correlations between fruit $\mathrm{pH}$ with FD, LBL and
LBW, PL, FL, FW, SW, and TSS (Table V). As depicted in Table $\mathrm{V}$, the percentage of TA had significantly negative correlations with tree height, TSS and $\mathrm{pH}$, while the correlation of TA with FD, LBL and LW, FL and FVW and FW/SW was significantly positive.

\section{F. Variability structure of 20 Afghan apricot cultivars}

The dendrogram based on UPGMA analysis or Unweighted Pair Group Mean Analysis showed two distinct clusters (I and II) (Fig. 1). The cluster I consisted of 13 genotypes while the cluster II comprised only of 7 genotypes. In addition, the clusters I and II were divided into two groups (IA and IB) and (IIA and IIB), respectively. IA group included six genotypes ("Shakarpara", "Walayati", "Waziri', "Shakarpara-Sorkh", "Shakarpara-Wakili", "Shirin-Bano") and IB group seven genotypes ("Koorpa", "Maidani", "Seyed-Abadi”, "Saqi”, "Bid-Mushk", "Gibraelli", "Baghal-Sorkh") and group IIA consisted of three genotypes ("Qadak", "Salari-Sorkh", "Hassan-Khili") and group IIB had four genotypes ("Pir-Naqshi", "Timuri", "Senjidi", "Amiri"). As shown in the Fig.1, the IA group was split into IA-1 and IA-2 subgroups, with five genotypes (("Shakarpara", "Walayati”, "Waziri', "Shakarpara-Sorkh", "Shakarpara-Wakili) in IA-1 and one genotype "ShirinBano" in IA-2.

Also, in the Cluster I, the IB group was further subdivided into IB-1 and IB-2 subgroups, with two genotypes ((“Koorpa", "Maidani”) in IB-1 and five genotypes ("SeyedAbadi”, "Saqi”, "Bid-Mushk", "Gibraelli”, "Baghal-Sorkh”) in IB-2. Additionally, in cluster II, group IIA was separated into IIA-1 and IIA-2 and IIB into IIB-1 and IIB-2 subgroups. In the IIA-1 subgroup two genotypes ("Qandak", "Salari-Sorkh"), in the IIA-2 subgroup one genotype "Hassan-Khili", in the IIB-1 subgroup three genotypes ("Pir-Naqshi", "Timuri", "Senjidi") and in the IIB-2 subgroup one genotype "Amiri". Genetic distances were short, and more similarities were observed in cultivars that included the IA-1 subgroup, while genetic distances were higher among cultivars included in the second cluster and more similarities were observed (Fig. 1). 


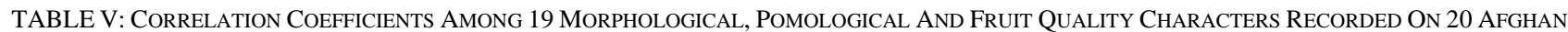
APRICOT CULTIVARS

\begin{tabular}{|c|c|c|c|c|c|c|c|c|c|c|c|c|c|c|c|c|c|c|}
\hline & $\mathrm{TH}$ & 1 & 2 & 3 & 4 & 5 & 6 & 7 & 8 & 9 & 10 & 11 & 12 & 13 & 14 & 15 & 16 & 17 \\
\hline $1-\mathrm{FD}$ & -0.188 & 1.000 & & & & & & & & & & & & & & & & \\
\hline 2-LBL & -0.105 & $.347^{* * *}$ & 1.000 & & & & & & & & & & & & & & & \\
\hline 3-LBW & 0.020 & 0.214 & $0.832^{* *}$ & 1.000 & & & & & & & & & & & & & & \\
\hline 4-LBL/LBW & -0.196 & 0.133 & $0.205-$ & $0.342^{* *}$ & 1.000 & & & & & & & & & & & & & \\
\hline 5-PL & 0.028 & 0.119 & $0.796{ }^{* * *}$ & $0.758^{* *}$ & -0.020 & 1.000 & & & & & & & & & & & & \\
\hline 6-LBL/PL & -0.2130 & $0.331^{* *}$ & 0.186 & 0.036 & $0.314 *$ & $0.419^{* *}$ & 1.000 & & & & & & & & & & & \\
\hline 7-PT & -0.148 & $0.288^{*}$ & 0.079 & 0.247 & $-0.294^{*}$ & -0.050 & 0.227 & 1.000 & & & & & & & & & & \\
\hline 8-FL & $-0.369^{* *} 0$ & $0.565^{* *}$ & 0.036 & -0.067 & 0.124 & -0.166 & $0.345^{* *}$ & 0.191 & 1.000 & & & & & & & & & \\
\hline 9-FVW & 0.025 & $0.328^{*}$ & $\mathrm{0.333}^{* *}$ & $0.333^{* *}$ & -0.065 & $0.278^{*}$ & 0.024 & 0.184 & 0.153 & 1.000 & & & & & & & & \\
\hline 10-FLW & -0.088 & 0.040 & -0.089 & -0.030 & -0.118 & -0.002 & -0.147 & 0.197 & $0.299^{*}$ & 0.161 & 1.000 & & & & & & & \\
\hline 11-FW & $-0.447^{* *}$ & $.358^{* * *}$ & 0.213 & 0.183 & -0.046 & 0.168 & 0.025 & -0.041 & $0.371^{* * *}$ & 0.254 & 0.247 & 1.000 & & & & & & \\
\hline 12-FSI & -0.007 & -0.212 & 0.179 & $0.255^{*}$ & -0.158 & $0.362^{* *}-($ & $-0.353^{* *}$ & -0.135 & $-0.562^{* * *}$ & 0.157 & 0.221 & $0.471^{* *}$ & 1.000 & & & & & \\
\hline $13-\mathrm{SW}$ & -0.162 & $0.285^{*}$ & $0.281^{*}$ & 0.215 & -0.039 & $0.266^{*}$ & -0.044 & 0.058 & 0.195 & $0.282^{*}$ & -0.030 & $0.512^{* *}$ & 0.225 & 1.000 & & & & \\
\hline 14-FW/SW & $-0.322^{*}$ & 0.165 & 0.008 & 0.020 & -0.020 & -0.025 & 0.049 & -0.051 & 0.218 & -0.022 & $0.257^{*}$ & $0.635^{* *}$ & $0.303^{*}$ & $-0.306^{*}$ & 1.000 & & & \\
\hline 15-MP & $-0.364^{* *}$ & 0.160 & 0.108 & 0.124 & 0.013 & 0.050 & 0.089 & -0.108 & 0.235 & 0.056 & $0.281^{*}$ & $0.556^{* *}$ & 0.253 & $-0.373^{* *}$ & $0.907^{* *}$ & 1.000 & & \\
\hline 16-TSS & $0.299^{*}$ & -0.129 & -0.112 & -0.118 & 0.019 & -0.142 & -0.023 & -0.039 & 0.001 & 0.022 & 0.141 & $-0.267^{*}$ & -0.152 & $-0.354^{* *}$ & -0.050 & 0.091 & 1.000 & \\
\hline 17-pH & $0.227-$ & $-0.316^{*}$ & $-0.417^{* *}$ & $-0.317^{*}$ & $-0.106-$ & $0.333^{* *}$ & -0.109 & -0.237 & $-0.304^{*}$ & 0.070 & -0.170 & $-0.271^{*}$ & -0.009 & $-0.356^{* *}$ & -0.013 & -0.001 & $0.307^{*}$ & 1.000 \\
\hline 18-TA & $-0.301 * 0$ & $0.337^{* *}$ & $0.664^{* *}$ & $0.570^{* *}$ & 0.091 & $0.382^{* *}$ & $0.372^{* * *}$ & 0.180 & $0.287^{*}$ & $0.382^{* *}$ & -0.027 & $0.298^{*}$ & 0.014 & $0.298^{*}$ & 0.087 & 0.128 & $-0.285^{*}$ & $-0.353^{* *}$ \\
\hline
\end{tabular}

$*$ and $* *$ show significant $(P \leq 0.05)$ and highly significant $(P<0.01)$ correlations, respectively.

As displayed in Fig.1, genetic distances of genotypes in cluster I on subgroup level were lower than on individual level in cluster II which in turn reveal higher similarities amongst cluster I genotypes than genotypes including in cluster II.

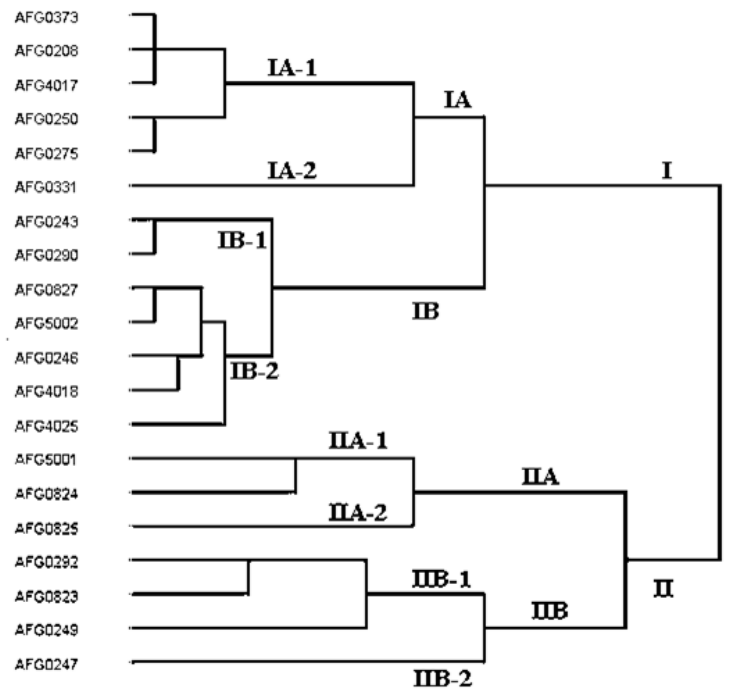

Fig. 1. Hierarchical clustering of 20 Afghan apricot genotypes based on 19 morphological, pomological and fruit quality traits using Euclidian distance with standard deviation.

\section{G. Principal component analysis (PCA)}

Principal component analysis was performed to identify the principal factors that explain the variability. PCA showed approximately $86.15 \%$ of total variance among 20 genotypes explained by the first seven components with higher than one eigenvalues (Table VI). The PC1, PC2 and PC3 components were responsible for $26.37 \%, 16.53 \%$ and $12.75 \%$ of variance, respectively. The most important morphological and pomological variables identified in the analysis of the principal components of the factors were LBL, LBW, PL, TA, FL, FLW, FW/SW ratio, FD, PW, FSI and LBL/LBW ratio. The main loading factors in PC1 were LBL and LBW, PL, TA and fruit $\mathrm{pH}$; and in PC2 were FL, FLW, FW/SW ratio, FD while in case of PC3 the loading factors were FSI and LBL/LBW ratio (Table VI).

TABLE VI: Eigenvalues, Variance Percentage, Cumulative VARIANCE PERCENTAGE AND LOAdING FACTORS FOR SEVEN First PRINCIPAL COMPONENTS (PC) FROM 19 MORPHOLOGICAL AND POMOLOGICAL VARIABLES OF 20 AFGHAN APRICOT GENOTYPES

\begin{tabular}{|c|c|c|c|c|c|c|c|}
\hline & \multicolumn{7}{|c|}{ Component loadings } \\
\hline & PC1 & $\mathrm{PC} 2$ & $\mathrm{PC} 3$ & PC4 & PC5 & PC6 & $\mathrm{PC} 7$ \\
\hline Tree height & -.003 & -.330 & -.120 & -.545 & -.436 & -.267 & .213 \\
\hline Flower diameter & .228 & .570 & .143 & .210 & -.035 & .305 & .442 \\
\hline Fruit length & -.118 & .772 & .144 & .261 & .258 & 194 & .255 \\
\hline Fruit lateral width & -.091 & 683 & -.155 & -.166 & .231 & -.334 & .079 \\
\hline Fruit ventral width & .269 & 109 & -.088 & -.056 & .030 & .108 & .881 \\
\hline Stone weight & .170 & .091 & .016 & .753 & -.224 & -.203 & .472 \\
\hline $\begin{array}{l}\text { Fruit:Stone weight } \\
\text { ratio }\end{array}$ & .046 & 699 & .098 & -.085 & 203 & 289 & -.055 \\
\hline TSS & -.100 & .181 & .093 & -.775 & .006 & -.178 & .112 \\
\hline Fruit weight & .106 & .318 & -.054 & .573 & .599 & -.133 & .364 \\
\hline TA & .626 & .035 & .028 & .319 & .201 & .517 & .304 \\
\hline $\mathrm{pH}$ & -.512 & -.446 & -.217 & -.483 & .156 & -.042 & .250 \\
\hline Leaf blade length & .959 & .047 & .135 & .081 & .030 & .109 & 166 \\
\hline $\begin{array}{l}\text { Leaf blade width } \\
\text { Leaf blade }\end{array}$ & .904 & -.019 & -.374 & .030 & .059 & .092 & .131 \\
\hline $\begin{array}{l}\text { length:Blade width } \\
\text { ratio }\end{array}$ & -.011 & .058 & .977 & -.008 & -.018 & .088 & -.026 \\
\hline $\begin{array}{l}\text { Petiole length } \\
\text { Leaf blade }\end{array}$ & .914 & -.068 & .033 & .076 & .038 & -.337 & .079 \\
\hline $\begin{array}{l}\text { length:petiole length } \\
\text { ratio }\end{array}$ & -.064 & .228 & .126 & .052 & .024 & .872 & .086 \\
\hline Petiole width & .122 & .556 & -.496 & .011 & -.421 & .329 & -.020 \\
\hline Mesocarp percentage & .124 & .263 & -.041 & -.119 & .917 & .069 & -.029 \\
\hline Fruit shape index & -.009 & .053 & .982 & -.014 & -.022 & .101 & -.031 \\
\hline Eigenvalue & 5.011 & 3.142 & 2.423 & 1.694 & 1.644 & 1.305 & 1.146 \\
\hline Variance (\%) & 26.377 & 16.537 & 12.755 & 8.920 & 8.657 & 6.873 & 6.034 \\
\hline $\begin{array}{l}\text { Cumulative variance } \\
(\%)\end{array}$ & 26.377 & 42.915 & 55.670 & 64.5907 & 73.248 & 80.121 & 86.155 \\
\hline
\end{tabular}




\section{DISCUSSION}

Favorable climactic and eco-geographical conditions pave the way for variations in plants so Afghanistan has rich diversity of apricot. However, but in case of absence of appropriate identification, characterization, documentation, management, competition from other high return fruit crops such as apple and burgeoning climate change, the apricot diversity in the country is under unknown and unprecedented threat.

As presented by some researchers namely Asma and Ozturk [6] and Badenes et al. [24], studying the distinct characters and traits of various pomological/botanical varieties is of immense importance and accounts a prerequisite for breeding and classification programs. This study showed high variability in different morphological characters among apricot varieties currently grown in Afghanistan. Highly polymorphic characters exist for apricot accessions.

\section{A. Variation in leaf size}

Great leaf size variations were found in this study and they might be partly due to variability in environment such as soil conditions, climate, and other salient features of the area. Leaf size may be considered as a significant indicator in identification of plants and also in photosynthetic activity which subsequently results in growth. These findings are in harmony with those reported by Perez-Gonzalez [31], who has showed strong correlation between fruit weight and growth habit of either bud size or leaf size.

Leaf is very important part of plant which serves as source of food to the plant and plays a vital role in plant growth and fruit development. In previous studies significant diversity in leaves and flowers of rosaceous fruits were reported. Environmental as well as genetic factors are responsible for the diversity and growth of plant species. Leaf area and their specific mass were found highly correlated with light intensity received. Garriz et al., [27] reported significant difference in leaf area having $18.31 \mathrm{~cm}$ in shade and $21.98 \mathrm{~cm}$ in light.

\section{B. Fruit Shape}

Fruit shape and color made important indices for marketability and customer appealing. Fruit shapes of apricot cultivars were different from each other. In the present study the predominant fruit shape and skin color were round and yellow green, respectively. The results of present study are closely related to fruit shapes of IranoCaucasian apricots group studied by Asma and Ozturk [6] and Yilmaz. et al., [17]. Thompson et al., [22] in a study found that cross pollination might be responsible to cause variability in morphological traits, especially in fruit shapes. Krichen et al. [18] reported round fruit shape and yellow to white skin color in Tunisian and Turkish apricots. These findings confirm that in terms of fruit shape and skin color, there is similarity among Turkish, Tunisian, and Afghan apricot cultivars. Krichen et al. [18] have been shown similar variability in fruit size of Tunisian apricot cultivars. Fruit shape has leading role in market values and is an important physical feature in fruit grading, sorting, packaging and transportation [9].

\section{Fruit Weight (g)}

Fruit weight plays significant role in yield and fruit quality as well as generates consumer preference. The fruit weight varied from minimum (18.17 g) in accession Qandak to maximum $(57.57 \mathrm{~g})$ in accession Shakarpara-Sorkh with $36.32 \mathrm{~g}$ mean weight. There identified two groups of apricot accessions based on fruit weight; small fruit $(<35 \mathrm{~g})$ and large fruit $(>36 \mathrm{~g})$. Ten genotypes had large fruits and ten others had small fruits. Such morphological variations in apricot fruits also reported by Kazankaya [1], Sharma et al., [10] and Bhat et al., [26].

Large size of fruit might be the genetic character of a cultivar to consume the available nutrients competently to attain a certain fruit size [8]. Trees with heavy crop load result in small fruit size and vice versa so thinning fruits in initial growth stages may favor large size fruits [11]. Asma and Ozturk [6] also have been found similar results in apricots of Irano-Caucasian group.

Fruit size is considered important trait to select the superior genotypes in apricot [25]. The space between plants to plant of same genus as well as other species can also affect the weight of fruit and size due to competition for sun light, water and nutrition uptake and also increases chances of diseases and insects attack. Low radiation and stress condition by water in plants decreases the supply of nutrients (cabohydrates) available for fruit enlargement and resulted in small size and weight of fruit. [15], [23].

\section{Correlations}

Significant correlations were found for the pomological characteristics of the studied cultivars/genotypes. The correlation between fruit size or weight and fruit length was 0.381 , while the correlation between fruit weight and flower diameter was 0.358 . The correlation between fruit weight and stone weight was 0.512 and between fruit weight and mesocarp percentage was 0.556 . These could be justified as fruit weight is directly related to fruit length, flower diameter, fruit flesh (mesocarp percentage) and stone weight. The correlation between fruit weight and ratio of fruit to stone weight was 0.635 , since larger fruits harbor larger stones. A negative correlation was obtained between fruit weight and total soluble solids $(-0.267)$, and between fruit length and fruit shape index $(-0.562)$. The greater the fruit size, the lower the concentration of soluble solids into fruit. Also as length of the fruit increases, the fruit shape becomes longer.

\section{E. Mesocarp percentage}

The edible portion or mesocarp of the fruit, computed as mesocarp percentage in this study, is an important pomological character which has unique and leading role in consumer preference and industry like juice extraction and canning. High mesocarp percentage representing desired attribute of table consuming apricot fruits. In this study, the fruits of accession Hassan-Khili had the highest mesocarp percentage (94.44\%) and fruits of accession Waziri had the lowest mesocarp percentage $(80.88 \%)$. This pomological attribute can be combined with other traits by breeding programs for introducing commercial apricot cultivars. 


\section{F. Kernel weight and taste}

Greater variability was found in stone weights of 20 Afghan apricot cultivars ranged from $4.37 \mathrm{~g}$ to $1.74 \mathrm{~g}$ (i.e. $4.37 \mathrm{~g}$ in Waziri and $1.74 \mathrm{~g}$ in Walayati). Apricot kernels are used as same as almond kernels and their interest has increased in recent years. Among studied apricot genotypes only one accession Walayati had bitter taste, while the kernels of remaining 19 cultivars were with absent or very weak bitterness. Fortunately, although the Walayati accession had bitter kernels, their kernel weight was the smallest too. Large and sweet kernels are generally consumed directly while the bitter ones are used in pharmaceutical and cosmetics. Aside from their utilization in direct consumption, drugs, and cosmetics the apricot kernels have also been used in genotype identification [21], [30].

\section{G. PCA and Dendrogram}

Principal component analysis uses to identify data patterns and to highlight similarities and differences [3], [32]. Also, the PCA has been applied for assessing correlations among tree, fruit and phonological attributes in apricot accessions/genotypes to display genetic relationships among accessions [24], [12], [28].

In this study approximately $86.15 \%$ of the total variance among 20 studied genotypes, was explained by the first seven components. The PC1, PC2, and PC3 accounted for about $26.37 \%, 16.53 \%$, and $12.75 \%$ respectively, of the total variability. Factors mainly caused high variation in 20 studied genotypes were length and width of the leaf blade, length of the petiole, fruit $\mathrm{pH}$ and titratable acidity, length and lateral width of the fruit, ratio between fruit weight and stone weight, flower diameter, fruit shape index, and ratio between blade length and width.

Multivariate analysis is an important statistical tool for identification of similar or closely related objects based on a set of shared attributes. In the current study, data generated from 20 apricot cultivars/genotypes was used for construction of dendrogram in order to assess the relatedness amongst the cultivars. The cluster analysis based on morphological and fruit quality traits generated two main clusters. The cluster I comprised of 13 cultivars/genotypes while the cluster II comprised of only seven cultivars/genotypes. The cluster I is further sub-divided into several sub-clusters based on different pomological and morphological attributes. Interestingly accession "Amiri" (AFG0247) formed a separate out group among all cultivars/genotypes included in the dendrogram. The fruit characteristics of this cultivar in terms of size, shape and weight were also found quite distinct from most of the cultivars. It seems that among all native cultivars accession "Amiri" is well adapted to the local climate and has the potential for use in future apricot breeding programs.

\section{CONCLUSIONS}

This is the first study conducted in Afghanistan to investigate genetic variability of apricot cultivars based on some fruit, leaf and other phonological, quality and quantity characters. Results showed high genetic variations among Afghan apricot cultivars. These variations provide better opportunities for Afghan growers to take advantage of the studied characters according to climatic conditions of growing areas. Also these variations pave the way for apricot breeders to introduce genotypes with elite traits and develop varieties adaptable to various agro-climatic conditions.

\section{ACKNOWLEDGMENT}

We highly acknowledge the contribution of Mr. Etemadi (field horticulturist in Badambagh research station, Kabul) and Mr. Ahadi (fruit germplasm curator, Kabul) in relation to providing the data and financial support of ministry of agriculture, irrigation and livestock (MAIL) of Afghanistan. Thanks also extend to Mr. Samim for critical revision of the manuscript.

\section{REFERENCES}

[1] A. Kazankaya, "Pomological traits of apricots (Prunus armeniaca L.) selected from Bitlis seedling population," J. Am. Pomol. Soc., vol. 56, pp. 184-188, 2002.

[2] A. Zanetto, L. Maggioni, R.K. Tobutt and R. Dosba, "Prunus genetic resources in Europe: Achievement and perspectives of a networking activity,". Genetic Res. and Crop Evol., vol. 49, pp. 331-337, 2002.

[3] A.L. Mattos, P.E. Amorim, O.B.V. Amorim, O.K. Cohen, S.A.C Lodo, and S.O. Silva, "Agronomical and Molecular Characterization of Banana Germplasm,” Pesq. Agrop. Bras., vol. 45, pp. 146-154, 2010 .

[4] B. Shirzad, and G.R. Samadi, Deciduous Fruits' Production in Afghanistan. $2^{\text {nd }}$. ed. Kabul, Afghanistan: Kabul University Publications, 2018, chap. 5, pp. 93-118.

[5] B. Shirzad, Deciduous Fruits' Production in Afghanistan, $1^{\text {st }}$ ed. Kabul, Afghanistan: Kabul University Publications, 1994, chap. 5, pp. 75-95.

[6] B.M. Asma, and K. Özturk, "Analysis of morphological, pomological and yield characteristics of some apricot germplasm in Turkey,' Genet. Res. Crop Evol, vol. 52, pp. 305-313, 2005.

[7] B.M. Asma, T. Kan, and O. Birhanli, "Characterization of promising apricot (Prunus armeniaca L.) genetic resources in Malatya, Turkey,' Genet. Res. Crop. Evol. vol.54, pp. 205-212, 2007.

[8] C.J. Stanley, D.S. Tustin, G.B. Lupton, S. McArthney, W.M. Cashmore, and H.N. De Silva, "Toward understanding the role of temperature in apple fruit growth responses in three geographical regions within New Zealand,". J. Hort. Sci. \& Biotech., vol. 5, pp. 413-422, 2000.

[9] D. Erdogan, M. Guner, E. Dursun, and I. Gezer, "Mechanical harvesting apricots," Biosyst. Eng., vol. 85, pp. 19-28, 2003.

[10] D.P. Sharma, N. Sharma, R. Bawa, and K. Rajesh, "Potential of apricot growing in the arid-cold desert region of North-Western Himalayas," Acta Hortic., vol. 696, pp. 61-63, 2005.

[11] E. Lotze, and O. Bergh, "Early prediction of harvest fruit size distribution of an apple and pear cultivar," Scientia Hortic., vol.101, pp. 281-290, 2004.

[12] F. Gurrieri, J.M. Audergon, G. Albagnac, and M. Reich, 2001 "Soluble Sugars and Carboxylic Acids in Ripe Apricot Fruit as Parameters for Distinguishing Different Cultivars," Euphytica, vol. 117, pp. 183-189, 2001.

[13] FAOSTAT, 2017. Agricultural Production, Crops Primary. http://apps.fao.org/faostat.

[14] G., Ozkan, and M.A. Koyuncu, "Pysical and chemical composition of some walnut (Juglans regia L.) selection grown in Turkey," Grasus Aceites, vol. 56, pp. 141-146, 2005.

[15] H.W. Caspari, M.H. Behboudian, and D. G. Chalmers, "Water use growth, and fruit yield of 'Housi' Asian pears under deficit irrigation," J. Amer. Soc. Hortic. Sci., vol. 119, pp. 383-388, 1994.

[16] J.M. Audergon, M. Souty, J.M. Duffillol, and M. Breulls, "Biochemical and physico-chemical characterization of 400 apricot varieties consequences in the apricot selection and improvement process," Acta Hortic, vol. 293, pp. 111-119, 1991.

[17] K.U. Yilmaz, S. Paydas-Kargi, Y. Dogan and S. Kafkas, "Genetic diversity analysis based on ISSR, RAPD and SSR among Turkish Apricot Germplasms in Iran Caucasian eco-geographical group," Sci. Hort., vol. 138, pp. 138-143, 2012. 
[18] L. Krichen, J.M. Audergon, and N. Trifi-Farah, "Variability of morphological characters among Tunisian apricot germplasm." Sci. Hort., vol. 179, pp. 328-339, 2014.

[19] M. Faust, D. Suranyl, and F. Nyujto, "Origin and dissemination of apricot," Hort. Rev., vol. 22, pp. 225-266, 1998.

[20] M. Faust, S. Dezsö, and N. Ferenc, "Origin and Dissemination of Prunus Crops: Peach, Cherry, Apricot, Plum and Almond," ISHS (Int. Soc. Hortic. Sci.). Hortic. Rev., vol. 22, pp. 103- 138. Wiley, 2011.

[21] M. Özcan, "Composition of Some Apricot Prunus armeniaca Kernels Grown in Turkey," Acta Aliment., vol. 29, pp. 289-294, 2000.

[22] M.D. Thompson, R. Brenner, Z. Anwar, and S. Bhatti, Biogeographic survey and collection of temperate fruit and nut genetic resources in Northern Pakistan. p. 105, 1988.

[23] M.H.G. Behboudian, S. Lawes, and K.M. Griffiths, "The influence of water deficit on water relations, photosynthesis and fruit growth in Asian pear (Pyrus serotina Rehd.),". Sci. Hortic., vol. 60, pp. 89-99, 1994.

[24] M.L. Badenes, J. Martinez-Calvo, and G. Llacer, "Analysis of apricot germplasm from the European ecogeographical group," Euphytica, vol. 102, pp. 93-99, 1998.

[25] M.N. Westwood, and L.T. Blaney, "Non-climatic factors affecting the shape of apple fruits," Nature, vol. 200, pp. 802-803, 1963.

[26] M.Y. Bhat, B.A. Padder, A. Wani, A. Imtiyaz, F.A. Banday, H Ahsan, M.A. Dar, and A.A. Lone, "Evaluation of apricot cultivars based on physicochemical characteristics observed under temperate conditions," Int. J. Agric. Sci., vol. 3, pp. 535-537, 2013.

[27] P.I. Garriz, G.M. Colavita, and H.L. Alvarez, "Fruit and spur leaf growth and quality as influenced by low irradiance levels in pear," Scientia Hortic., vol. 77, pp. 195-205, 1998

[28] R. Azodanlou, C. Darbellay, J.L. Luisier, J.C. Villettaz, and R. Amadò, "Development of a Model for Quality Assessment of Tomatoes and Apricots," Food Sci. Technol, vol. 36, pp. 223-233, 2003.

[29] R. Guerriero F. Lomi and C. D'onofrio, "Influence of some agronomic and ecological factors on the constancy of expression of some descriptive characters included in the UPOV apricot descriptor list," Acta Hortic., vol.717, pp. 51-54, 2006.

[30] S. Mandal, S. Poonam, S.K. Malik, and S.K. Mishra, "Variability in Kernel Oil, Its Fatty Acid and Protein Contents of Different Apricot (Prunus armeniaca) Genotypes," Indian J. Agr. Sci., vol.77, pp. 464466, 2007.

[31] S. Perez-Gonales, "Association among morphological and phenological characters representing apricot germplasm in central Mexico," J. Amer. Soc. Hort. Sci., vol. 117, pp. 486-490, 1992.

[32] T. Milosevic, and N. Milosevic, "Genetic Variability and Selection in Natural Populations of Vineyard Peach (Prunus persica ssp. vulgaris Mill.) in the Krusevac Region (Central Serbia)," Agrociencia, vol. 44, pp. 297-309, 2010

[33] T. Miloševic, N. Miloševic, I. Glišic, and I.S. Glišic, I.S. 2014 "Determination of size and shape properties of apricots using multivariate analysis," Acta Sci. Pol., Hortorum Cultus, vol. 13, pp. 77-90, 2014.

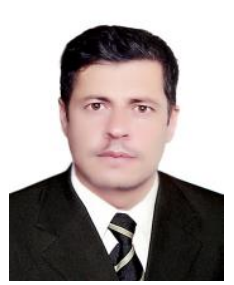

Hedayatullah Salari is currently working as associate professor, horticulture department, agriculture faculty, Paktia university. He has been working there for more than 14 years. He was born in 1984 in Afghanistan. He earned his BSc in General Horticulture from Kabul University in 2007 and MSc in Pomology from Isfahan University of Technology (IUT) in 2013, Isfahan, Iran. He has worked with AHNDO (Afghan National Horticulture Development Organization) as research program officer and data analyst. He analyzed more than 25 research projects replicated each one for 3 years. He guided and accomplished about 10 research projects. He has about 15 published papers (in nation and international journals).

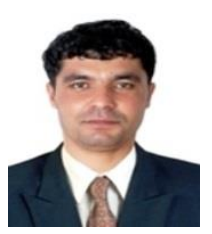

Alam Khan Samim is currently working as assistan professor, horticulture department, agriculture faculty, Paktia University. He has been working there for more than 8 years. He was born in 1985 in Afghanistan. He earned his BSc in General Horticulture from Paktia University in 2010 and MSc in Pomology from University of Agricultural and Horticultural Sciences, Shivamogga (UAHS, Shivamogga) in 2018, Karnataka, India. He guided and accomplished about 6 research projects. He has about 5 published papers (in nation and international journals). 